\title{
About the new internal symmetries of the Lattice $Z^{\mathrm{N}}$
}

\author{
Kornyushkin A* \\ Moscow Institute of Physics and Technology, Russia
}

*Corresponding author: Kornyushkin A, Moscow Institute of Physics and Technology, Russia

\section{Introduction}

A large (apparently, infinite!) number of internal symmetries of the lattice $\mathrm{Z}^{\mathrm{N}}$ is identified. Their presence stems from one interesting observation. It concerns Cellular Automation. If we take a secondorder reversible Automata [1] in its simplest version (the so-called Fredkin's Simplified Automata whose behaviour depends solely on the shape of a certain neighbourhood in $\mathrm{Z}^{\mathrm{N}}$ ), and run it under two certain conditions (arbitrary and arbitrary -"transliterated"), then they will continue going completely "symmetrical", all the way, from the Point of Origin to the Point of the Mirror. (What exactly is this "symmetry" is described in detail in [2-7]). This property is performed for some neighbourhoods (or as we write - "Masks") (we call such neighbourhoods Perfect), and for others - no (not Perfect). (Figure 1). The paradox of the situation is that there are a lot of Perfect neighbourhoods! For example, seven Perfect neighbourhoods with their properties are reflected in Appendix 1. The analysis of this feature leads to the fact that we can "get rid" of the Cellular Automation and come to very interesting structures, the so-called Transition Tables (TT) [4-6]. (The Cellular Automation needs only to build the TT themselves, then they begin to "live their lives"). We have counted a total of 8(!) properties of TT, some of which are very paradoxical. We will list them with conventional names along with the short commentary:

\section{Perfect neighborhood Unperfect neighborhood $(19,11)$ One dimensional case \\ $(21,11)$

One dimensional case

Figure 1.

a) «Substitutions». (Transition tables are six tables made up of six-digit numbers. The number of columns is equal to the number of cells in the Mask. The number of rows $(R)$ is some basic constant for each Mask. All 5 other Transition Tables are obtained from the first Table by corresponding substitutions). b) «Graph». (For our proof, a graph composed of TT with nodes at Mask cells must satisfy some simple property).

c) "Closed Lotto game". (The main paradoxical property! Can be formulated as an independent integer colouring problem. Our TT miraculously solve it!)

d) "Constant accurate calculation". (Another completely new paradoxical property! Just like property 3 , it can be easily checked on the computer).

e) «Inverse transformation». (One additional TT property which clearly has some binding nature).

f) "Division of TT rows into two parts; "right" and "left"”. (Reference to the third property. The corresponding diagram clearly shows the division).

g) "The main law of conservation". (Back to where we started - to Cellular Automation! For any single CA, empirically find a law of conservation: $\mathrm{w}_{0}(\tau)+\mathrm{w}_{1}(\tau)=\mathrm{w}_{1}(\tau+1)-\mathrm{w}_{0}(\tau+1)$. Here $\mathrm{w}_{0}$ and $w_{1}$ are the number of cells in the Automata obtained from the "left" and "right" rows summed throughout the Automata; $\tau$ is the time) [7].

h) There are related lectures on the «Alex Kornushkun» YouTube channel. Today there are 5 of them. We are preparing the sixth. Please pay attention to the first Lecture and the program key_5m3.exe (the link is given in the description of the Lectures). The program (along with the Lecture itself) reproduces a step-by-step proof of the Perfection of this Mask. Note how computer proof leads us to the desired result "in the happiest way"! Clearly, this can't just be a coincidence.

\section{References}

1. Toffoli T, Margolus N (1987) Cellular Automaton Machines: A New Environment for Modeling, MIT Press Series, Section 14.2, Second-order technique pp. 147-149.

2. Wolfram Stephen (2002) A New Kind of Science, Wolfram Media, pp. 437. 
3. McIntosh HV (2009) “One Dimensional Cellular Automaton”, Luniver Press, pp. 205-246.

4. Kornyushkin A (2017) Strict Proof of the Perfection of the First 98 Masks (Solution to the "X-Problem of the Number 3") Int J Swarm Intel Evol Comput 6: 2.

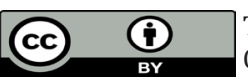

This work is licensed under Creative Commons Attribution 4.0 License

To Submit Your Article Click Here:

Submit Article

DOI: $10.32474 /$ CTCSA.2019.01.000114
5. Kornyushkin A (2016) Theory of mathematical strings: the first steps. Int J Inf Res Rev 3(9): 2764 - 2783.

6. Kornyushkin A The X-problem of number 3.

7. Kornyushkin A About a Discrete Cellular Soliton (computer simulation).

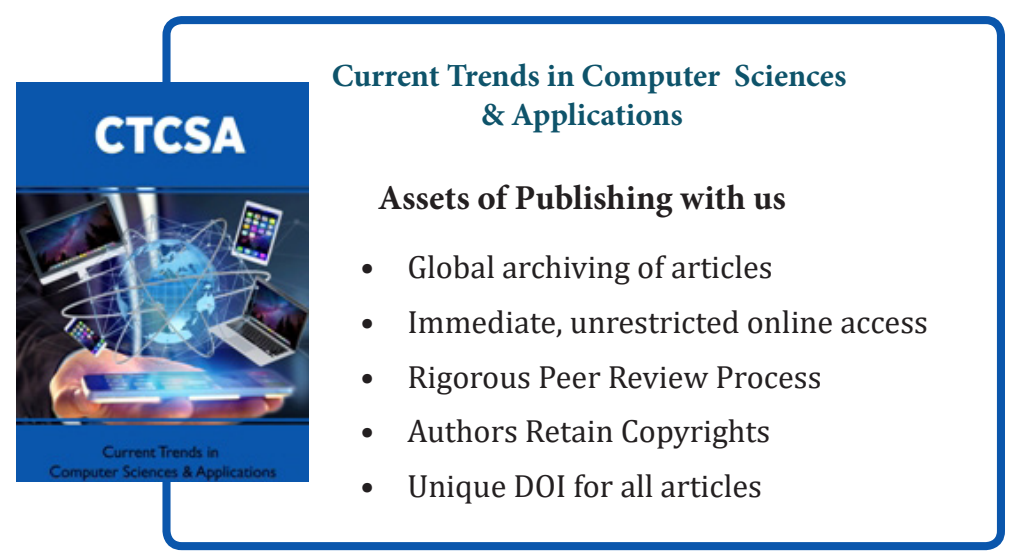

